\title{
Intracellular Spatial Control of Fluorescent Magnetic Nanoparticles
}

\author{
Jinhao Gao, Wei Zhang, Pingbo Huang, Bei Zhang, Xixiang Zhang and Bing Xu ${ }^{*}$
}

\section{Supporting Information}

Chemicals. Oleic acid (99\%), oleylamine (99\%), phenyl ether (99\%), trioctylphosphine (TOP, 99\%), glutathione (GSH), selenium powder (99.98\%), 1, 2-hexadecandiol (tech 90\%), Fe(acac) 3 (98\%), and $\mathrm{CdO}$ (99\%) were purchased from Sigma-Aldrich. All the chemical reactions were carried out under the inert atmosphere in a glove box unless otherwise stated.

Instrumentation. The transmission electron micrographs (TEM) were obtained on a JEOL 2010 electron microscopes operated at $200 \mathrm{kV}$, the high-resolution TEM (HRTEM) images and energy dispersive X-ray spectrometer (EDS) on a JEOL 2010F high resolution electron microscope operated at $200 \mathrm{kV}$, the absorbance and fluorescence spectra on a Perkin Elmer Lambda 900 UV/VIS/NIR spectrometer and a Perkin Elmer luminescence spectrometer (LS55), respectively, and the confocal images on a confocal laser scanning microscope (Leica Microsystems, Wetzlar, Germany).

Synthesis of 4-nm $\mathrm{Fe}_{3} \mathrm{O}_{4}$ nanoparticles. ${ }^{1} 1 \mathrm{mmol}$ of $\mathrm{Fe}(\mathrm{acac})_{3}, 5 \mathrm{mmol}$ of 1, 2-hexadecandiol, 3 mmol of oleic acid, $3 \mathrm{mmol}$ of oleylamine and $15 \mathrm{~mL}$ of phenyl ether were mixed and heated to reflux for $0.5 \mathrm{hr}$ in a glove box with an argon atmosphere. After cooled down to the room temperature, the $\mathrm{Fe}_{3} \mathrm{O}_{4}$ nanoparticles were washed with ethanol and redissolved in hexane.

Synthesis of $\mathbf{F e}_{3} \mathrm{O}_{4}$-CdSe nanoparticles. ${ }^{2} \sim 20 \mathrm{mg}$ of the as-prepared $\mathrm{Fe}_{3} \mathrm{O}_{4}$ nanoparticles, 0.5 mmol of $\mathrm{CdO}$ powder and $15 \mathrm{~mL}$ of phenyl ether were mixed and heated to reflux for $10 \mathrm{~min}$. After cooled down to $220^{\circ} \mathrm{C}$, a selenium stock solution ( $1 \mathrm{mmol}$ of Se powder dissolved in $2 \mathrm{~mL}$ of TOP) was dropped into the solution slowly ( $\sim 3 \mathrm{~min})$. After addition, the nanoparticles grew at $250{ }^{\circ} \mathrm{C}$ for about $10 \mathrm{~min}$ to reach the desired size. Then, the $\mathrm{Fe}_{3} \mathrm{O}_{4}-\mathrm{CdSe}$ nanoparticles were washed with ethanol and redissolved in hexane to form a stable dispersion.

Quantum yield. The quantum yield of $\mathrm{Fe}_{3} \mathrm{O}_{4}-\mathrm{CdSe}$ heterodimer nanoparticles was measured in hexane using Rhodamine $6 \mathrm{G}$ as the standard (quantum yield $=95 \%)^{3}$

Surface modification of $\mathrm{Fe}_{3} \mathrm{O}_{4}$-CdSe nanoparticles using GSH. Under the ambient condition, 1 $\mathrm{mL}$ of hexane solution containing $\sim 10 \mathrm{mg}$ of $\mathrm{Fe}_{3} \mathrm{O}_{4}-\mathrm{CdSe}$ nanoparticles was added into $1 \mathrm{~mL}$ of the water solution containing $\sim 2 \mathrm{mg}$ of GSH (adjusted $\mathrm{pH} \sim 7$ ). These two solutions were mixed by vigorous stirring and kept stirring for about 24 hours in order to let the $\mathrm{Fe}_{3} \mathrm{O}_{4}$-CdSe nanoparticles completely transfer from the hexane layer into the water layer. The $\mathrm{Fe}_{3} \mathrm{O}_{4}-\mathrm{CdSe} @ \mathrm{GSH}$ sample was 
dispersed in water and attracted onto the wall of the vial by a small magnet, and the supernatant was discarded. The nanoparticles were purified by repeating the above procedures two more times. In the end, the attracted nanoparticles were redispersed in water very well.

Cell transfection and confocal imaging. HEK293T cells were cultured in Dulbecco's Modified Eagle's Medium (DMEM) and placed into 24-wells for transfection. pEGFP vector together with the $\mathrm{Fe}_{3} \mathrm{O}_{4}-\mathrm{CdSe} @$ GSH nanoparticles were transfected into cells by a lipofectamine 2000 (Invitrogen) treatment. After 24 hours, the solution was replaced and washed by fresh DMEM and the cells were placed onto cover slides. Then a small magnet was fixed under the slide (one slide without the magnet as the control experiment). After 8 hours, the cells were used for the confocal imaging. Green fluorescence of the GFP and red fluorescence of the nanoparticles were excited with two different and fast alternated beams at $488 \mathrm{~nm}$ and $543 \mathrm{~nm}$ to avoid cross talk. The fluorescence emission imaging was collected in two separate channels at $532 \pm 56 \mathrm{~nm}$ and $645 \pm 65$ $\mathrm{nm}$, respectively. All the resulting images were acquired, stored and visualized with the Leica confocal software (LCS).

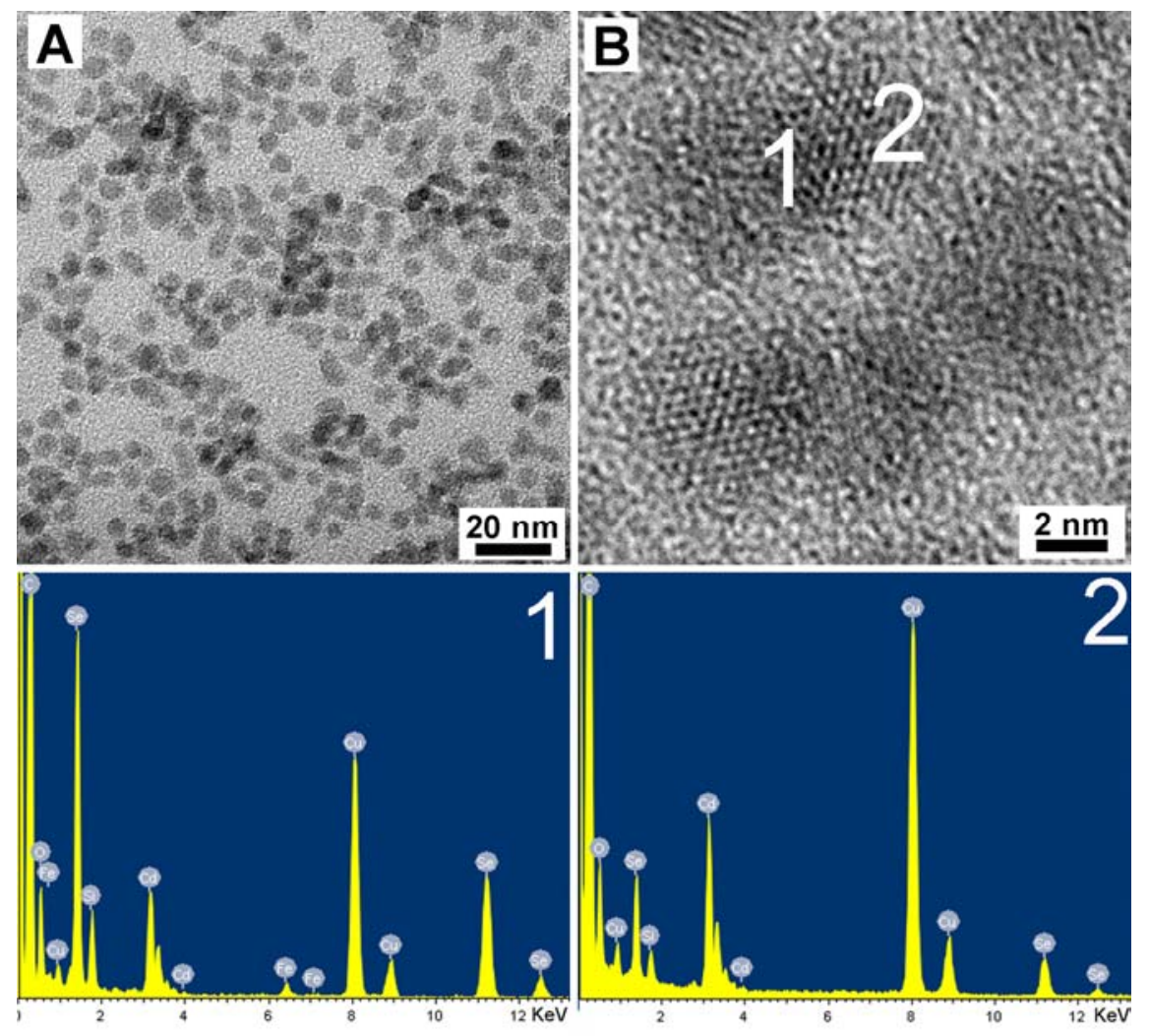

Figure S1. (A) The TEM and (B) HRTEM images of $\mathrm{Fe}_{3} \mathrm{O}_{4}-\mathrm{CdSe}$ nanoparticles. The EDS analysis of the $\mathrm{Fe}_{3} \mathrm{O}_{4}$ part and $\mathrm{CdSe}$ part, corresponding to the regions 1 and 2 in the HRTEM image, respectively. The EDS results indicated the $\mathrm{Fe}_{3} \mathrm{O}_{4}$ nanoparticle part may be coated with a thin layer of CdSe. 

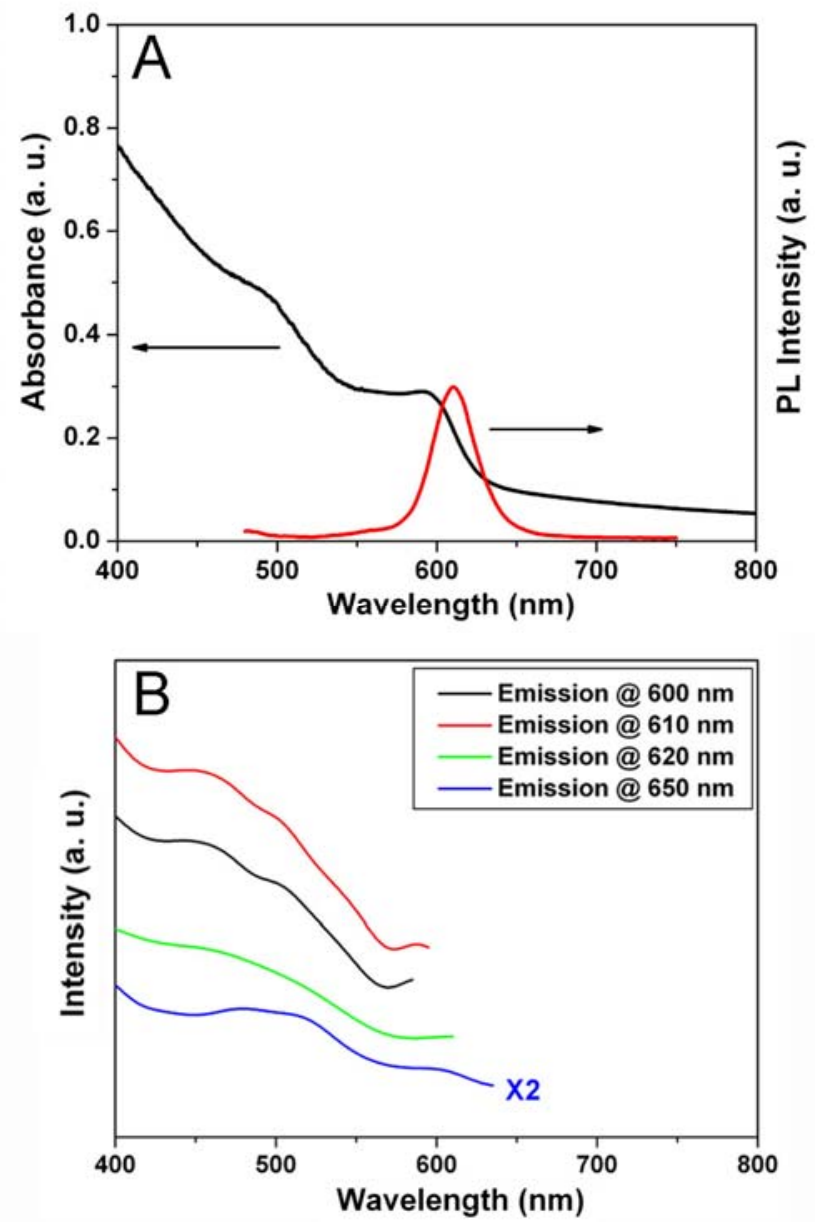

Figure S2. (A) The UV-vis and fluorescence spectra of $\mathrm{Fe}_{3} \mathrm{O}_{4}-\mathrm{CdSe}$ nanoparticles. (B) The photoluminescence excitation (PLE) spectra of $\mathrm{Fe}_{3} \mathrm{O}_{4}-\mathrm{CdSe}$ nanoparticles under the different emission wavelengths.

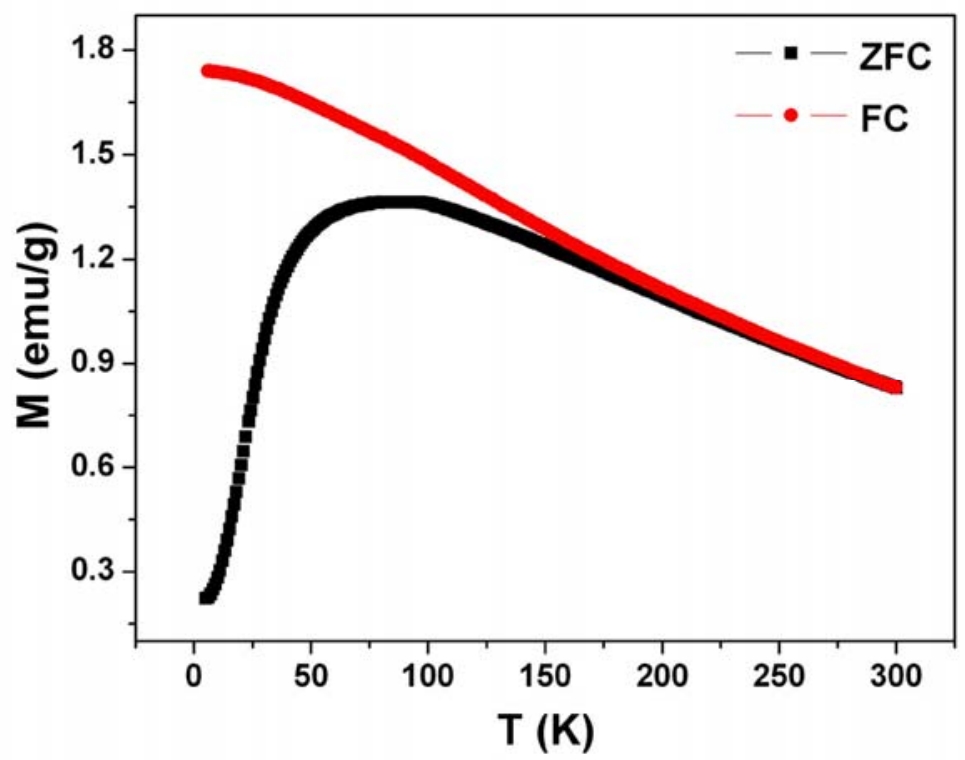

Figure S3. The temperature-dependent of the $\mathrm{ZFC} / \mathrm{FC}$ magnetization curves of $\mathrm{Fe}_{3} \mathrm{O}_{4}-\mathrm{CdSe}$ nanoparticles with a magnetic field of 100 Oe. 


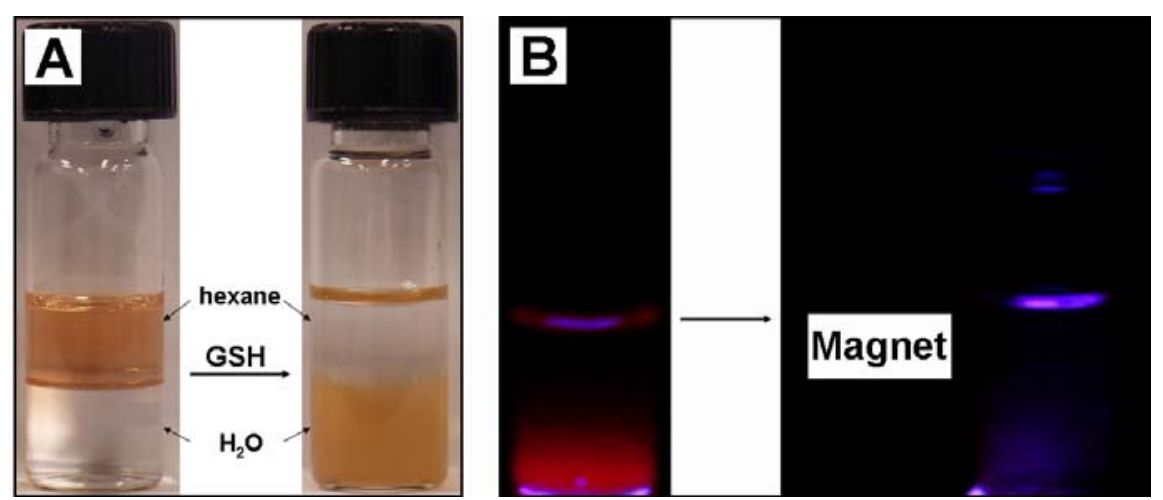

Figure S4. (A) The Optical images of $\mathrm{Fe}_{3} \mathrm{O}_{4}-\mathrm{CdSe}$ nanoparticles transferred from hexane layer to water layer after surface modification by glutathione (GSH) molecules. (B) The fluorescence images of water solution of $\mathrm{Fe}_{3} \mathrm{O}_{4}-\mathrm{CdSe} @ \mathrm{GSH}$ nanoparticles before and after attracted by a small magnet (excitated by a UV lamp, $\lambda_{\mathrm{ex}}=365 \mathrm{~nm}$ ).

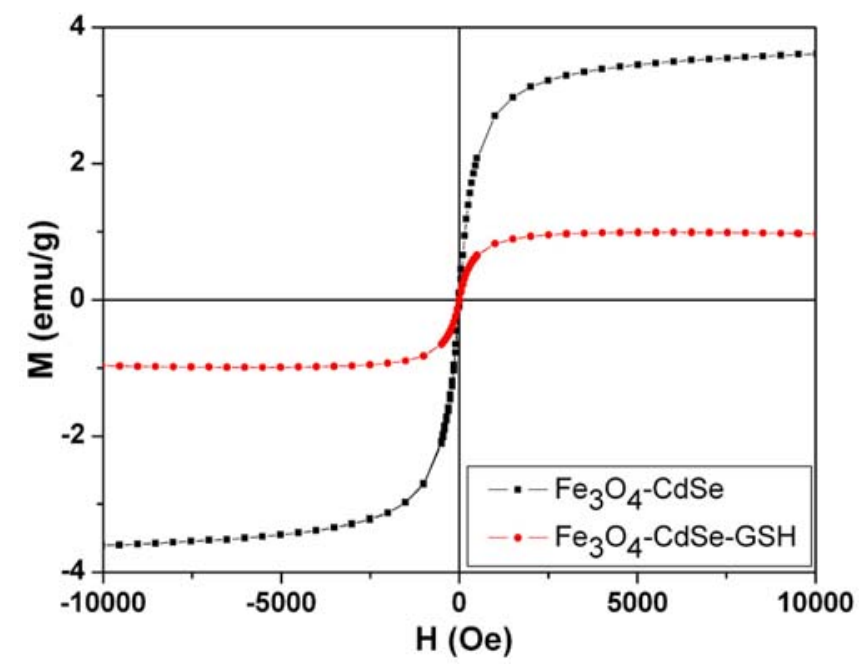

Figure S5. The hysteresis loops obtained at $300 \mathrm{~K}$ for $\mathrm{Fe}_{3} \mathrm{O}_{4}-\mathrm{CdSe}$ nanoparticles and $\mathrm{GSH}$ coated $\mathrm{Fe}_{3} \mathrm{O}_{4}-\mathrm{CdSe}$ nanoparticles.

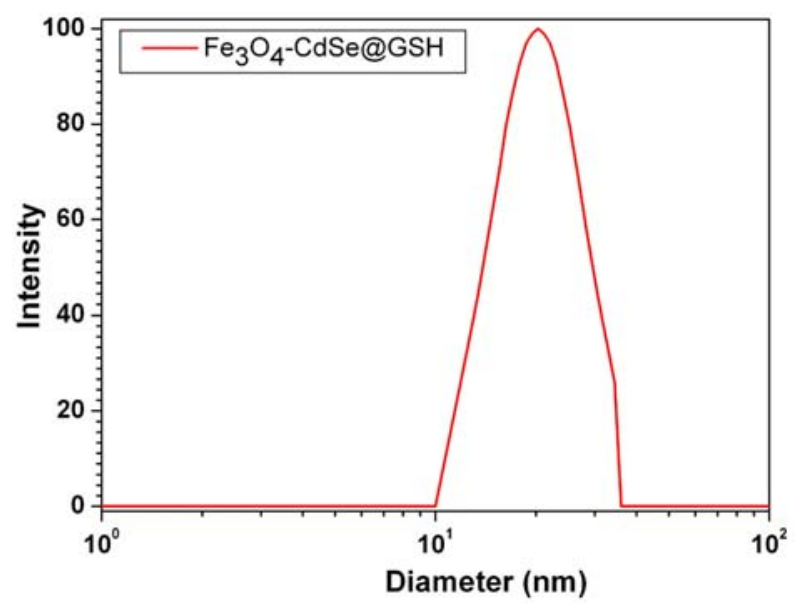

Figure S6. Dynamic light scattering (DLS) data of $\mathrm{Fe}_{3} \mathrm{O}_{4}-\mathrm{CdSe} @ \mathrm{GSH}$ nanoparticles. 


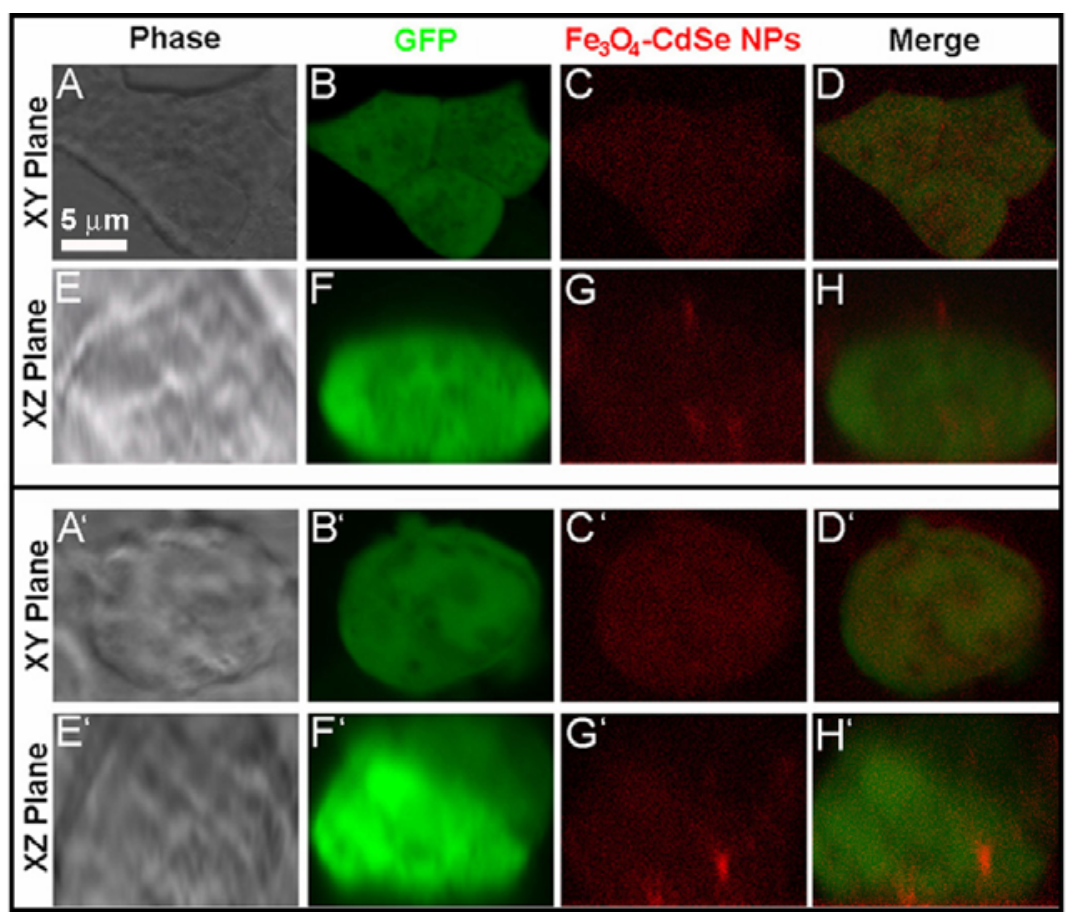

Figure S7. Two sets of typical confocal images taken from control experiments (without a magnetic field) by XY plane (A,A', phase; B,B', GFP vector dyed; C,C', cellular uptake of $\mathrm{Fe}_{3} \mathrm{O}_{4}-\mathrm{CdSe}$ nanoparticles; D,D', merge) and XZ plane (E,E', phase; F,F', GFP vector dyed; G, G', cellular uptake of $\mathrm{Fe}_{3} \mathrm{O}_{4}-\mathrm{CdSe} @ \mathrm{GSH}$ nanoparticles; H,H', merge).

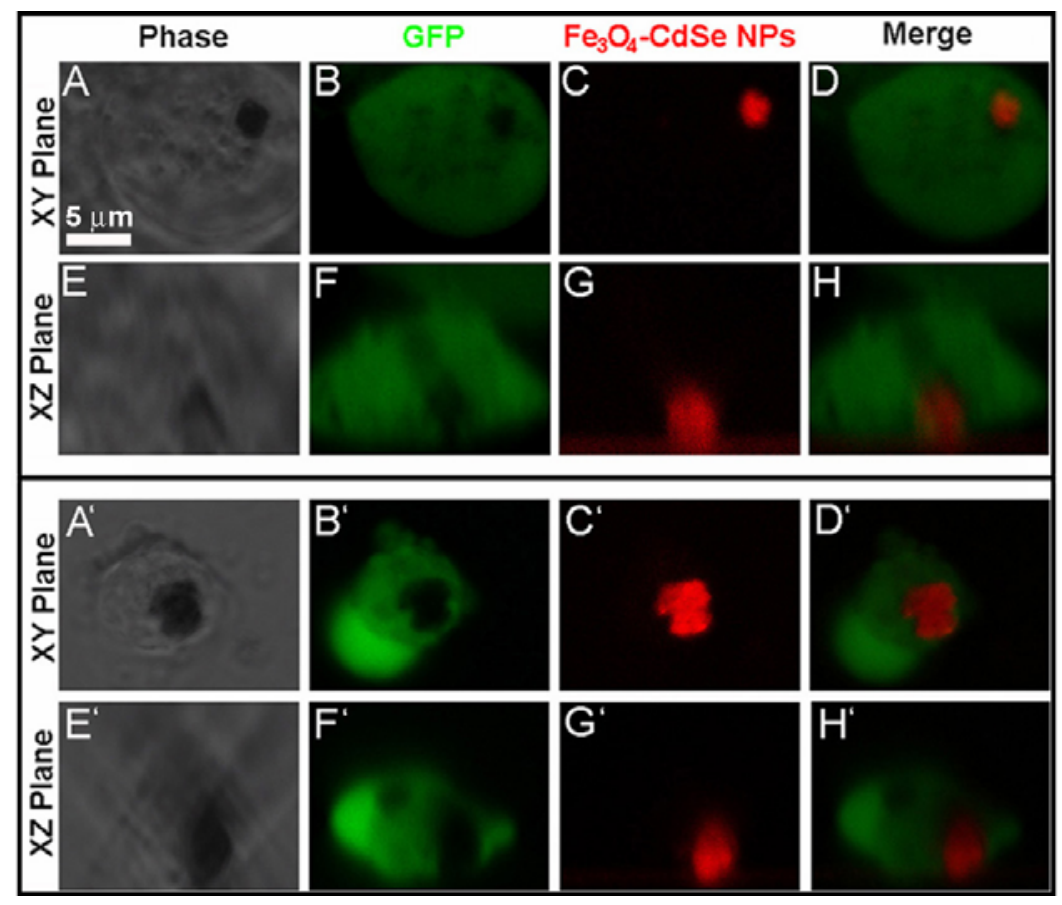

Figure S8. Two sets of the typical confocal images taken from the samples under a magnetic field (the magnet was fixed at the bottom of the samples) by XY plane (A,A', phase; B,B', GFP vector dyed; C,C', cellular uptake of $\mathrm{Fe}_{3} \mathrm{O}_{4}$-CdSe nanoparticles; D,D', merge) and $\mathrm{XZ}$ plane (E,E', phase; F,F', GFP vector dyed; G,G', cellular uptake of $\mathrm{Fe}_{3} \mathrm{O}_{4}$-CdSe@GSH nanoparticles; H,H', merge). 


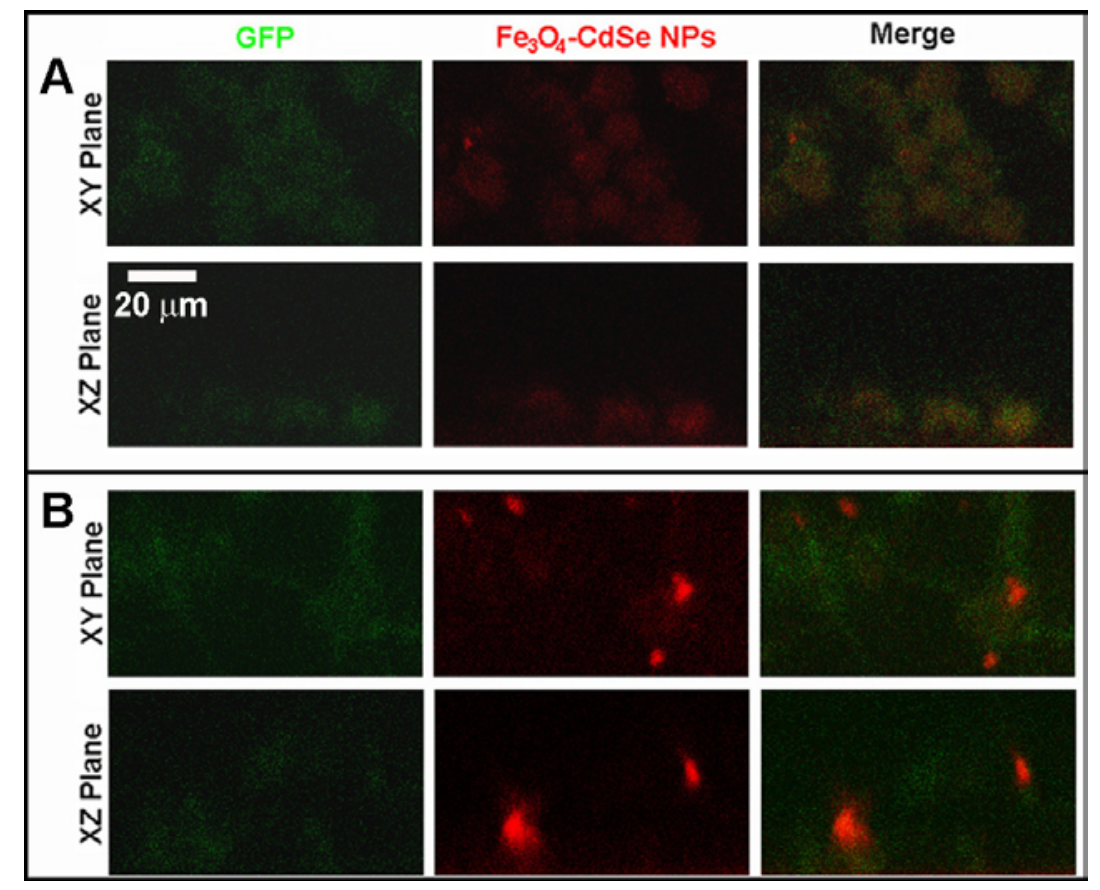

Figure S9. Low-magnification confocal images of the cells taken from the sample (A) without a magnetic field and (B) under a magnetic field (the magnet was fixed at the top of the sample). Almost all of the nanoparticles moved towards the small magnet and located the one side of the cells.

\section{References}

(1) Sun, S. H.; Zeng, H. J. Am. Chem. Soc. 2002, 124, 8204.

(2) Peng, Z. A.; Peng, X. G. J. Am. Chem. Soc. 2001, 123, 183.

(3) Bao, H. B.; Gong, Y. J.; Li, Z.; Gao, M. Y. Chem. Mater. 2004, 16, 3853. 